\title{
Priming by motion too rapid to be consciously seen
}

\author{
Uwe Mattler \\ Georg-August-Universität Göttingen, Göttingen, Germany \\ AND \\ ROBERT FENDRICH \\ Otto-von-Guericke-Universität Magdeburg, Magdeburg, Germany
}

\begin{abstract}
When a rapidly rotating ring of dots was briefly flashed, observers saw only a solid ring with no discriminable rotation. However, when this stimulus served as a prime that was followed by a target that consisted of a clearly rotating ring of dots, response times (RTs) to report the target's rotation were shorter when the prime and target directions were congruent than when they were incongruent. In accord with shape priming data, this priming effect increased monotonically with the prime-target stimulus-onset asynchrony (SOA). The prime also biased the perceived direction of an ambiguous apparent motion target, but only at an intermediate SOA. At the same SOA, we also found that target presentations enabled above-chance discrimination of prime's rotation direction. These outcomes demonstrate the processing of motion direction information that is not phenomenally represented. They suggest a common mechanism may mediate the priming of RTs by shape and motion, whereas a different mechanism mediates perceptual measures of motion priming.
\end{abstract}

One approach to understanding consciousness is to examine the brain's ability to process stimuli that do not reach awareness. In the visual domain, several studies have demonstrated that stimuli that observers do not consciously perceive can nevertheless serve as primes that alter the processing of subsequent visual stimuli (see, e.g., Eimer \& Schlaghecken, 1998; Fehrer \& Raab, 1962; Klotz \& Neumann, 1999; Lleras \& Enns, 2004; Marcel, 1983; Mattler, 2003, 2005, 2006; Milliken, Joordens, Merikle, \& Seiffert, 1998; Neumann \& Klotz, 1994; Schmidt, 2000, 2002; Vorberg, Mattler, Heinecke, Schmidt, \& Schwarzbach, 2003, 2004; Wolff, 1989). With shape and color stimuli, priming is normally demonstrated by the effect of the primes on the choice response time (RT) to respond to subsequent targets: RTs are often reduced when the prime and target are congruent (associated to the same response) than when they are incongruent (associated with different responses). This is the case even when primes are rendered invisible by backward masking by the subsequent target (Fehrer \& Raab, 1962; Klotz \& Neumann, 1999; Mattler, 2003; Neumann \& Klotz, 1994; Schmidt, 2000, 2002; Vorberg et al., 2003, 2004; Wolff, 1989). Motion priming has been demonstrated with a different type of measure: The direction of a moving prime will bias the perceived direction of a subsequent ambiguous apparent motion target (Anstis \& Ramachandran, 1987; Blake, Ahlstrom, \& Alais, 1999; Pantle, Gallogly, \& Piehler, 2000; Piehler \& Pantle, 2001; Pinkus \& Pantle, 1997; Ramachandran
\& Anstis, 1983). We will refer to this biasing effect as disambiguation. Blake et al. (1999) demonstrated motion priming by a stimulus outside awareness by showing that disambiguation priming can occur even when the prime is presented to an eye in which phenomenal vision is suppressed because of binocular rivalry.

These studies of priming by invisible stimuli have depended on processes that disrupt conscious awareness to make normally visible stimuli invisible. To understand the mechanisms of unconscious priming in such studies, one must know the level at which the disrupting process operates. For both masking (Breitmeyer, 1984; Macknik \& Livingstone, 1998; diLollo, Enns, \& Rensink, 2000) and rivalry (Blake, 2001; Blake \& Logothetis, 2002; Tong, 2001, 2003), this level has been disputed. However, several studies have demonstrated the processing of phenomenally invisible stimuli in a more direct manner. Research has shown that grating stimuli with spatial frequencies that are too high to be consciously represented can nevertheless generate tilt aftereffects (He \& MacLeod, 2001; Rajimehr, 2005) and produce distinguishable patterns of V1 activity (Haynes \& Rees, 2005). Research has also shown that in macaque monkeys, V1 cells respond to heterochromatic flicker at rates faster than the monkeys can discriminate (Gur \& Snodderly, 1997). In accord with this observation, Vul and MacLeod (2006) — using human subjects - found the color aftereffect that was described by McCollough (1965) when color alternations were too

U. Mattler, uwe.mattler@psych.uni-goettingen.de 
fast to be perceptible. Using achromatic flicker, Williams, Mechler, Gordon, Shapley, \& Hawken (2004) have found that the firing of some cells in human V1 can be entrained by high contrast video flicker at rates as rapid as $100 \mathrm{~Hz}$, well above the flicker fusion threshold. Because they do not depend on an external disrupting process to render stimuli invisible, studies of this kind provide a straightforward way of appraising the visual system's ability to process stimuli outside of conscious awareness.

To date, one study has addressed the issue of whether motion attributes that are intrinsically invisible can nevertheless be processed within visual cortex. Watanabe, Nanez, \& Sasaki (2001) showed that viewing undetectable levels of motion coherence can prime observers' detections of motion in the same direction in a subsequent stimulus. In the present study, we investigated the processing of intrinsically invisible motions with a very different type of stimulus. We looked for motion priming effects with a prime that consisted of a briefly flashed circle of 16 dots that rotated so rapidly, participants could not perceive its rotation direction. In one condition, this prime was followed by a target that consisted of a circle of 16 dots with a clearly visible clockwise or counterclockwise apparent rotation, and RTs to report the direction of the target rotation were recorded. This allowed us to assess priming with an RT measure of the sort previously used in studies of shape priming, so that if unconscious motion priming was found, we could compare its time course to the time course of the unconscious priming reported with shape stimuli. If these different forms of priming follow a similar time course as SOA is varied, this would be consistent with the hypothesis that the priming is mediated by a common system that receives input from both shape and motion stimuli. In another condition, the prime was followed by a target with an ambiguous rotation direction, and subjects reported the direction that was seen. This allowed us to evaluate the priming produced by our stimuli using the disambiguation measure that has been employed in most earlier motion priming studies (Anstis \& Ramachandran, 1987; Blake et al., 1999; Pantle et al., 2000; Piehler \& Pantle, 2001; Pinkus \& Pantle, 1997; Ramachandran \& Anstis, 1983).

\section{METHOD}

\section{Participants}

Ten students (ages 19 to 34, 7 female, 3 male) from the University of Magdeburg participated in the experiment. Nine were right handed by self-report, and all had normal or corrected-to-normal vision. Each participant received $€ 36$ for taking part in five 1.5 -h sessions, with each session run on a separate day.

\section{Display Properties}

The stimuli were presented on a Hewlett Packard 1310A X-Y display. Participants were seated in a dark room with their heads on a chinrest that was located $57 \mathrm{~cm}$ in front of this display. The HP display monitor is essentially a large screen oscilloscope (50-cm diagonal) that has been customized with a fast P15 phosphor (50 $\mu \mathrm{sec}$ luminance decay time to $0.1 \%$ ). Since we deemed that the use of the HP display with its fast refresh capability and ultra-fast phosphor was essential for producing the rapid motions we required, we were limited to the use of bright stimuli against a dark background. Both the prime and target stimuli always consisted of a set of 16 luminous dots (each dot about $3.5 \mathrm{~cd} / \mathrm{m}^{2}$ on a black background) arranged in a circle $5^{\circ}$ of visual angle in diameter with 1,440 potential display positions on its circumference. The dots were uniformly spaced around the circle with an angular separation of $22.5^{\circ}$ as shown in Figure 1A. The location of all 16 dots could be updated every millisecond, so that the effective "frame rate" was $1000 \mathrm{~Hz}$. A luminous fixation point was presented at the circle's center.

\section{Priming Stimulus}

The priming stimulus was identical in all the experimental runs (Figure 1B). Nineteen frames were presented at $1 \mathrm{frame} / \mathrm{msec}$, so that the total prime duration was $19 \mathrm{msec}$. On successive frames, each dot moved $1.25^{\circ}$ of angle clockwise or counterclockwise, reaching the position of its clockwise or counterclockwise neighbor in 18 steps, so that the first and final frames were identical. This produced an effective rotation rate of the entire circle of $1,250^{\circ} / \mathrm{sec}$. Since the linear distance that the dots moved on each step (about $3.25^{\prime}$ of arc) was smaller than their diameter (just under $4^{\prime}$ of arc), their successive positions overlapped, so that continuous motion was closely approximated. Due to visual persistence, the prime appeared phenomenally to be a briefly flashed unbroken outline circle.

\section{Conditions}

Every participant was used in four experimental conditions.

RT. In this condition, participants reported the rotation direction of an unambiguous apparent motion target that followed the prime in a speeded-choice RT task. The sequence of events is shown in Figure 2. The dots in the target display advanced clockwise or counterclockwise by steps of $7.5^{\circ}$ of angle every $133 \mathrm{msec}$ (Figure $1 \mathrm{C}$ ), producing an apparent rotation with a velocity of $56.4^{\circ} / \mathrm{sec}$. The dots remained visible at each location until they switched to the next location. Targets followed the prime with an SOA of 19, 44, 69,119 , and $219 \mathrm{msec}$, with each SOA occurring 100 times (in a random sequence) in a 500 -trial run. Since the prime duration was fixed at $19 \mathrm{msec}$, the prime-target ISIs associated with these SOAs were $0,25,50,100$, and $200 \mathrm{msec}$. On half of the trials at each SOA, the prime and target rotation directions were congruent (e.g., clockwise-clockwise), and on the other half, they were incongruent (e.g., clockwise-counterclockwise). Participants were instructed to indicate the direction in which the target was rotating as quickly and accurately as possible by pressing a button with their left (for counterclockwise) or right (for clockwise) index finger. A digital I/O port polled the response buttons every millisecond. After 2,400 $\mathrm{msec}$, the target display vanished, the fixation point blinked off briefly, and an audible beep signaled an error if the participant had not responded or reported the target's rotation incorrectly. The reappearance of the fixation point marked the beginning of the next trial. No mention was made of the prime. This condition was run first in two sessions, but the first session was treated as training and only data from the second session was analyzed.

Disambiguation. In this condition, participants reported the perceived direction of an ambiguous apparent motion target in an unspeeded task (see Figure 2). The dots in the target display shifted position by $11.25^{\circ}$ of angle every $200 \mathrm{msec}$ (Figure 1D). Since the step size was exactly half of the distance between the dots, the direction of the target's apparent rotation could be seen as either clockwise or counterclockwise, with an apparent velocity of $56.3 \% \mathrm{sec}$. Procedures were otherwise the same as those in the RT condition, except that error feedback was given only if the participant failed to respond. This condition was run in the third session.

Prime + discrimination. In this condition, participants judged the prime's rotation direction when the prime was followed by an unambiguous apparent motion target. The stimuli were, in all respects, identical to those employed in the RT condition, but participants were informed about the existence of the prime and asked to judge its rotation direction in an unspeeded task. They were instructed to guess the direction of the prime's rotation even if they did not consciously see it. An audible beep signaled either failures to respond within 
A

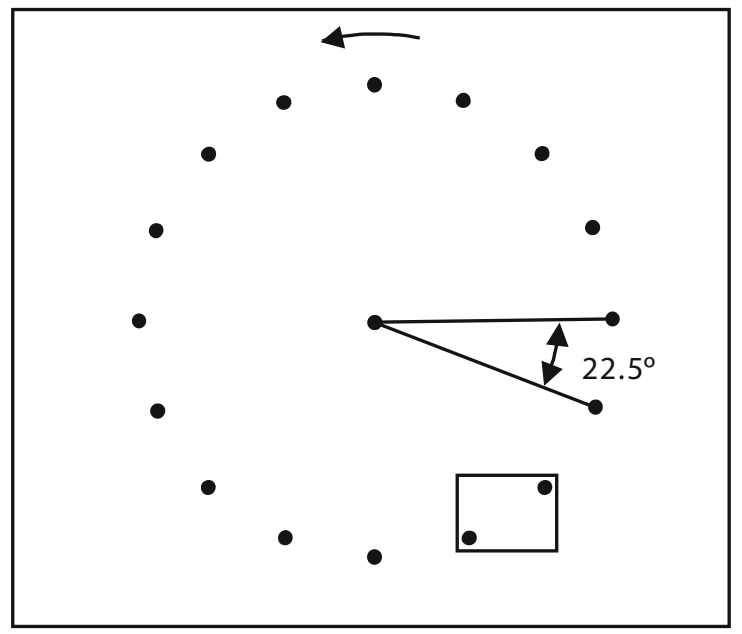

B

Prime
C

Apparent Motion Targets

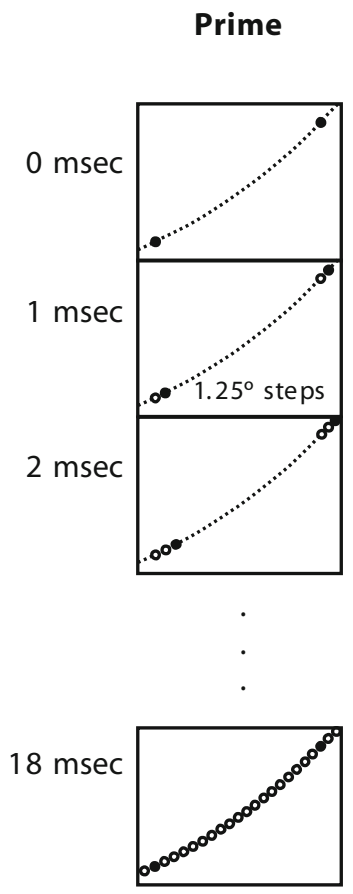

Directional

Ambiguous
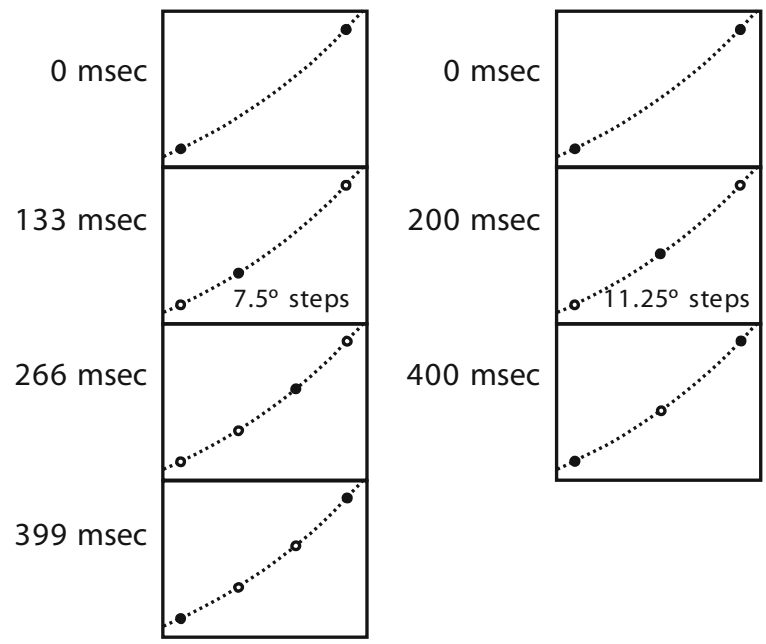

Figure 1. (A) The 16-dot circular display with the fixation point. The circle diameter was $5^{\circ}$. Counterclockwise rotation is illustrated, but the actual rotations could be either clockwise or counterclockwise. All 16 dots always moved in virtual tandem (within a $500 \mu$ s interval). The inset indicates the portion of the display illustrated in Figures 2B-2D. In these figures, black dots illustrate the actual dot positions in the display frame shown, and white dots show the dot positions in prior frames. (B) The frame sequence of the priming display. The apparent path of the dots is illustrated as a thin dotted line. Frames were updated every millisecond. Each $1.25^{\circ}$ angular position shift entailed a visual angle position change of $3.27^{\prime}$ of arc. (C) Successive frames of the target display in the RT and prime + discrimination conditions. Target dot positions advanced $1 / 3$ of the interdot distance $\left(7.5^{\circ}, 19.6^{\prime}\right.$ of visual angle) every $133 \mathrm{msec}$. (D) Successive frames of the target display in the disambiguation condition. Dots positions in the target display advanced by $1 / 2$ the interdot distance (11.25 of angle, $29.4^{\prime}$ of visual angle) every 200 msec.

2,400 msec or direction errors, providing participants with an opportunity to learn to use any subtle direction cues that were available.
Prime-only discrimination. In this condition, participants reported the prime's rotation direction when only the prime was pre- 


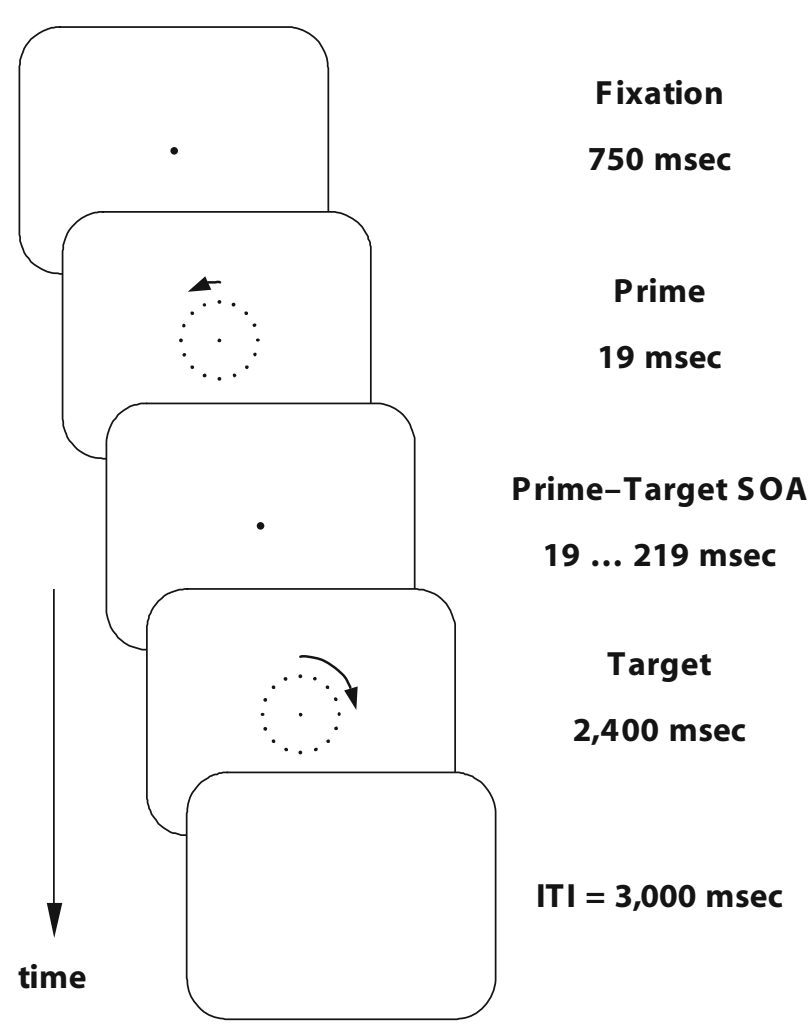

Figure 2. Schematic diagram of stimulus events. The prime was followed by an unambiguous (RT condition; the prime + discrimination condition) or ambiguous target display (disambiguation condition). Priming was assessed by RTs to report the target direction (RT condition) or prime-target direction congruency (disambiguation condition). In both cases, the prime-target stimulus onset asynchrony (SOA) was varied from trial to trial by adjusting the prime target ISI. In the prime recognition conditions, participants reported the direction of the prime's rotation, either when a target followed the prime (prime + discrimination condition) or when only the prime was presented (prime-only discrimination condition, not shown).

sented. They were allowed $2.4 \mathrm{sec}$ to respond to each presentation. One hundred trials were run in a random order, half with a counterclockwise rotation and half with a clockwise prime rotation. An audible beep signaled errors.

\section{Analysis Methods}

The RT data from each participant in the RT Condition were summarized by trimmed means, with error and posterror trials excluded, and were analyzed with a two-way repeated measures ANOVA, with SOA and congruency as factors. Error rates in the reports of target direction from this session were analyzed with a similar ANOVA performed on the arc-sine transformed mean proportion of incorrect direction responses. Congruency effects at specific SOAs were evaluated by $t$ tests. ANOVAs and $t$ tests were also used to evaluate the relationship between priming effects and prime discrimination performance. All reported ANOVA $p$ values were Geisser-Greenhouse corrected, but for readability, the uncorrected degrees of freedom are stated.

Pearson's $\chi^{2}$ tests, which are more sensitive than $t$ tests, were employed in order to evaluate the number of correct responses in the prime-only discrimination, prime + discrimination, and disambiguation conditions. Performance indices in these conditions were estimated separately for each participant and stimulus condition (e.g., motion direction of the target stimulus), and $\chi^{2}$ was calculated from the corresponding prime direction $\times$ response cross table. Global $\chi^{2}$ tests are based on the $\chi^{2}$ statistics summed across participants and stimulus conditions.

\section{RESULTS}

\section{Discrimination of the Prime Direction}

As previously indicated, the prime appeared to be a briefly flashed outline circle. When it was presented by itself (the prime-only discrimination condition), no participant could report at better than a chance level, whether its rotation was clockwise or counterclockwise. Averaged across participants, reports of the prime direction had a mean accuracy of $47.4 \%$ (95\% confidence interval $\left.44.3 \%-50.6 \%, \chi_{10}^{2}=7.63, p=.67\right)$. Table 1 gives the identification data separately for each participant.

Participants continued to be poor at discriminating the prime's rotation direction when a target with a clear apparent motion followed the prime (the prime + discrimination condition). Figure $3 \mathrm{~A}$ plots the percent-correct primeidentification performance at five prime-target SOAs (19, $44,69,119$, and $219 \mathrm{msec}$ ). Averaged across the five SOAs, prime-recognition responses were correct on $51.7 \%$ of the trials, which did not differ significantly from a chance value of $50 \%$ ( $95 \%$ confidence interval $44.3 \%-53.8 \%$, $\left.\chi_{10}^{2}=28.1, p=.11\right)$. However, when the performance at each level of SOA was examined, we found that when the SOA was $119 \mathrm{msec}$, prime-recognition performance was slightly but significantly better than chance $(55.2 \%$, $95 \%$ confidence interval $51.3 \%-59.1 \%, \chi_{20}^{2}=42.5, p=$ $.002)$. At all other levels of SOA, prime-recognition performance did not differ from chance $\left(\chi_{20}^{2} \leq 26.3, p>.15\right.$, in all cases). Table 1 gives the prime + discrimination data for the individual participants at each SOA.

\section{Priming Assessed With RT and Error Measures}

Although participants were completely unable to discriminate the prime's rotation direction in the primeonly condition, that direction had a pronounced effect on RTs to identify the direction that a subsequent target stimulus was rotating (the RT condition). Figure 3B plots the RT to identify the target direction at each of five prime-target SOAs for the congruent and incongruent prime-target pairings. ANOVA results show both the main effect of congruency $[F(1,9)=23.7, p=.001]$ and a significant congruency $\times$ SOA interaction $[F(4,36)=$ $32.4, p<.001]$. The main effect of congruency occurs because there is a significant RT reduction in congruent trials in comparison with incongruent trials at the three longest SOAs $[t(9)>2.9, p<.017$ in all cases]. The congruency $\times$ SOA interaction occurs because the positive congruency effect increases as SOA increases. This increase is monotonic, going from a negative value with the 19-msec SOA (shorter RTs when the prime and target motions were incongruent) to a peak at $119 \mathrm{msec}$ (see Figure 4). With each participant, we performed a linear regression analysis of the priming function between 19and $119-\mathrm{msec}$ SOA. The results of these analyses indicate that the priming function had a significantly negative 
Table 1

Prime Identification Performance in Different Conditions for Each Participant (Percent Correct)

\begin{tabular}{|c|c|c|c|c|c|c|c|c|c|c|c|}
\hline \multirow[b]{4}{*}{$\mathrm{P}$} & \multicolumn{11}{|c|}{ Condition } \\
\hline & \multirow[b]{3}{*}{ Prime Only } & \multicolumn{10}{|c|}{ Prime-Target SOA (msec) } \\
\hline & & \multicolumn{5}{|c|}{ Prime + Discrimination } & \multicolumn{5}{|c|}{ Disambiguation } \\
\hline & & 19 & 44 & 69 & 119 & 219 & 19 & 44 & 69 & 119 & 219 \\
\hline 1 & 52.0 & 52.6 & 53.6 & 54.5 & 58.0 & 46.9 & 55.0 & 56.1 & 55.7 & 63.0 & 45.8 \\
\hline 2 & 43.9 & 51.0 & 45.9 & 45.9 & 56.6 & 56.1 & 53.1 & 48.0 & 45.0 & 55.1 & 50.5 \\
\hline 3 & 40.8 & 49.5 & 42.3 & 53.1 & 50.0 & 49.5 & 49.0 & 54.1 & 45.9 & 58.2 & 48.5 \\
\hline 4 & 43.9 & 47.5 & 42.3 & 48.0 & 62.9 & 62.0 & 55.7 & 62.6 & 64.9 & 67.7 & 59.6 \\
\hline 5 & 45.9 & 49.0 & 49.0 & 46.4 & 53.0 & 41.7 & 49.5 & 48.5 & 50.0 & 50.5 & 50.0 \\
\hline 6 & 44.9 & 43.4 & 49.0 & 59.6 & 54.6 & 46.9 & 46.9 & 54.1 & 54.1 & 66.3 & 49.5 \\
\hline 7 & 52.0 & 53.5 & 54.5 & 60.8 & 60.8 & 55.6 & 52.0 & 48.5 & 54.1 & 77.0 & 66.7 \\
\hline 8 & 50.0 & 49.0 & 53.1 & 57.1 & 59.0 & 61.9 & 57.6 & 50.5 & 54.0 & 72.9 & 81.4 \\
\hline 9 & 54.1 & 55.6 & 53.1 & 56.7 & 44.9 & 40.4 & 32.7 & 34.3 & 32.0 & 42.7 & 41.8 \\
\hline 10 & 46.9 & 50.0 & 45.4 & 45.5 & 52.0 & 51.5 & 47.0 & 56.7 & 44.4 & 62.6 & 55.2 \\
\hline
\end{tabular}

Note-P, Participant; SOA, stimulus onset asynchrony between first and second stimuli (prime and target). See text for descriptions of conditions.

mean intercept $[-35.3 \mathrm{msec}, t(9)-4.9, p=.001]$ and, importantly, a slope that did not significantly differ from unity $[M=0.84, t(9)=-1.7, p=.12 ; 95 \%$ confidence interval 0.63-1.05].

When the prime and target were incongruent, there were significantly more errors than when they were congruent $[6.9 \%$ vs. $2.5 \%, F(1,9)=16.1, p=.003]$. Error rates for the congruent trials were generally flat in the vicinity of $2 \%$. Error rates for the incongruent trials increased as SOA increased from $0.2 \%$ at the 19 -msec SOA to $14.8 \%$ at the $119-\mathrm{msec} \mathrm{SOA}$, producing a significant congruency $\times$ SOA interaction $[F(4,36)=18.6, p<.001]$. The peak in errors mirrors the peak RT increase for these trials. The data, therefore, provide no indication that the priming effect on RTs was associated with a speed-accuracy tradeoff.

\section{Priming Assessed With Ambiguous Apparent Motion Targets}

A more restricted priming effect was found when the dependent measure was the perceived direction of an ambiguous apparent motion target stimulus (the disambiguation condition, see Figure 3C). Averaged across the five SOAs, the perception of the direction of rotation of the ambiguous target corresponded with the direction of the prime rotation in $53.5 \%$ of the trials $(95 \%$ confidence interval $\left.48.1 \%-59 \%, \chi_{10}^{2}=112.8, p<.001\right)$. There was a significant effect of SOA on priming $[F(4,36)=7.1$, $p=.005]$. When SOAs were $119 \mathrm{msec}$ and $219 \mathrm{msec}$, participants were significantly biased toward reporting the direction of the target's rotation as matching the prime's rotation direction. At the $119-\mathrm{msec} \mathrm{SOA}$, subjects were $61.6 \%$ correct $(95 \%$ confidence interval $54.2 \%-69 \%$, $\left.\chi_{10}^{2}=98.85, p<.001\right)$. At the 219 -msec SOA, they were $54.9 \%$ correct (95\% confidence interval $46.5 \%-63.3 \%$, $\left.\chi_{10}^{2}=60.92, p<.001\right)$. There was no significant effect at the shorter $\operatorname{SOAs}\left(\chi_{10}^{2} \leq 17.34, p>.06\right.$, in all cases $)$. We deem it noteworthy that the peak priming effect in the disambiguation condition and the only instance of nonchance performance in the prime discrimination condition occur at the same $119-\mathrm{msec}$ SOA. ${ }^{1}$

\section{Priming As a Function of Prime Identification Performance}

Since the priming effect with ambiguous targets and above-chance detection of the prime direction were both observed when SOA was $119 \mathrm{msec}$, we thought that these effects might be related. To assess the relationship between prime discrimination performance and the priming effects, we divided participants into two groups, one made up of the five participants with the best prime discrimination performance and the other five with the poorest performance. This was done once using the prime-only discrimination data, and again using the prime + discrimination data. The division into good and poor performers with the prime-discrimination data was made on the basis of participants' mean performance averaged across all the SOA levels.

When the groups were formed on the basis of primeonly discrimination data, the good prime discriminators had a mean discrimination accuracy of $51.1 \%$, and the poor prime discriminators had a mean discrimination accuracy of $43.9 \%$. The performance measures in the prime + discrimination condition, RT condition, and disambiguation condition were analyzed with two-way ANOVAs using these groups as a between-subjects factor and SOA as a within-subjects factor. In no case was there a significant main effect of group $[F(1,8)<2.5, p>.15$ in all cases] or significant group $\times$ SOA interaction $[F(4,32)<2.0$, $p>.16$ in all cases]. We therefore found no indication of a relationship between performance in the prime-only condition and any other performance measure.

Next, we compared the good and poor discrimination groups that were formed on the basis of the prime + discrimination data. The mean accuracy rates for these groups were $54.0 \%$ and $49.3 \%$, respectively. When we evaluated the accuracy rates of these groups at each of the SOAs, we found the poor prime-discriminator group performed at chance at every SOA $\left(\chi_{10}^{2} \leq 14.55, p>.14\right.$ in all cases). With the good prime-discriminator group, however, accuracy rates were significantly better than chance when the SOA was $119 \operatorname{msec}\left(\chi_{10}^{2}=27.93, p=.002\right)$ and 


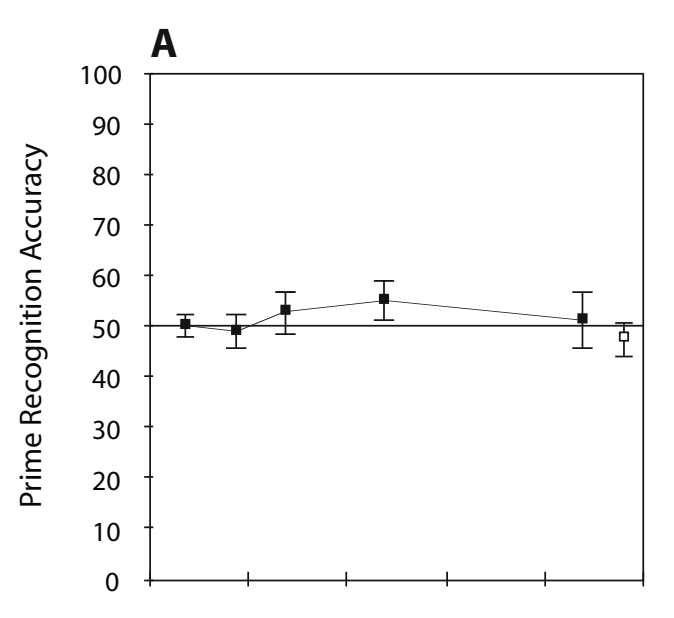

B
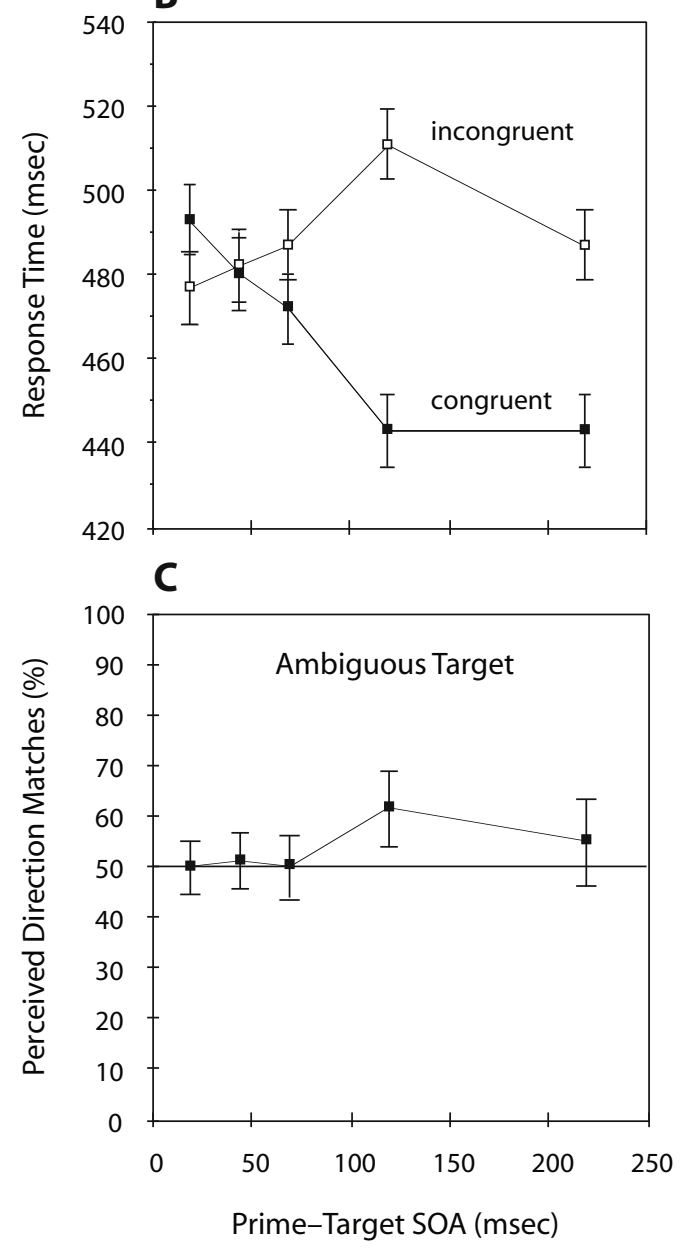

Figure 3. Prime recognition performance and priming effects as a function of the stimulus onset asynchrony (SOA). (A) Black symbols show prime discrimination performance in the prime + discrimination condition. The single white symbol shows the mean prime recognition performance in the prime-only discrimination condition in which there was no target. Bars show $95 \%$ confidence intervals. (B) Effects of congruent and incongruent primes on mean choice response time (RT) in the RT condition. Bars show 95\% confidence intervals of the congruency $\times$ SOA interaction (Loftus \& Masson, 1994). (C) Priming effects on the perceived rotation direction of the ambiguous target stimuli in the disambiguation condition. Bars show $95 \%$ confidence intervals.
$219 \operatorname{msec}\left(\chi_{10}^{2}=21.28, p=.019\right)$. At the other SOAs, performance did not deviate from chance $\left(\chi_{10}^{2}=11.98, p>\right.$ .28 in all cases). Figure 5A shows prime discrimination performance in both groups for each level of SOA.

We conducted two-way ANOVAs to evaluate the priming effects for these groups in the RT and disambiguation conditions, using group as a between-subjects factor and SOA as a within-subjects factor. In the RT condition, there was a significant main effect of group $[F(1,8)=$ $10.4, p=.012$ ], with a larger priming effect in the group with good prime + discrimination performance $(33 \mathrm{msec})$ than in the group with poor performance $(12 \mathrm{msec})$. The main effect of SOA was also significant $[F(4,32)=33.4$, $p<.001]$, reflecting the increase in priming to a peak at $119 \mathrm{msec}$. However, the interaction SOA $\times$ group did not reach significance $[F(4,32)=1.3, p=.31]$, indicating that the dynamic of the priming effect was not different in the two groups. In addition, a $t$ test of the slope of the priming function in the RT condition was not significantly different for the poor and good prime-discrimination groups $[t(8)=-1.3, p=.22]$, and in both groups, the slope of the priming function did not differ from that of unity $[t(4)=-1.9, p=.13$ for the poor discrimination group, $t(4)=-0.37, p=.73$ for the good group]. Individual $t$ tests at each level of SOA revealed significant negative priming in the poor prime-discriminator group at the 19 -msec SOA $[t(4)=-3.3, p=.029]$, and significant positive priming at the 119- and 219-msec SOAs $[t(4)>$ $5.9, p \leq .004$, in both cases]. In the group of good prime discriminators, significant priming was observed at the 119- and 219-msec SOAs $[t(4)>5.2, p \leq .006$, in both cases]. Figure 5B summarizes these results and shows a shift towards increased positive priming in the group with good prime discrimination performance at every SOA.

Figure $5 \mathrm{C}$ shows the performance in the disambiguation condition for the groups with poor and good prime + discrimination performance. Visual inspection suggests consistently stronger priming in the group with good prime discrimination performance, but the pattern of the data in the two groups appears to be similar. Statistical analyses support these observations. The main effect of group was marginally significant $[F(1,8)=5.3, p=.051]$, and the main effect of SOA was significant $[F(4,32)=6.7, p=$ $.007]$, but the SOA $\times$ group interaction did not approach significance $[F(4,32)=0.7, p=.49]$. With the poor prime-discrimination group, individual $\chi^{2}$ tests at each level of SOA revealed a significant priming effect with $119-$ msec SOA $\left[\chi_{5}^{2}=21.54, p<.001\right]$. With the good prime-discrimination group, significant priming effects occurred with SOAs at the 69-, 119-, and 219-msec SOAs $\left[\chi_{5}^{2} \geq 14.39, p<.02\right.$ in all cases $]$.

To sum up, individual differences in the prime discrimination performance assessed in the prime-only condition were not related to the observers' abilities to discriminate the prime direction when the prime was followed by the target stimulus (the prime + discrimination condition), or to the priming effects observed in the RT and disambiguation conditions. This is not surprising, given that no subject in the prime-alone condition performed significantly better than chance. On the other hand, when the prime 


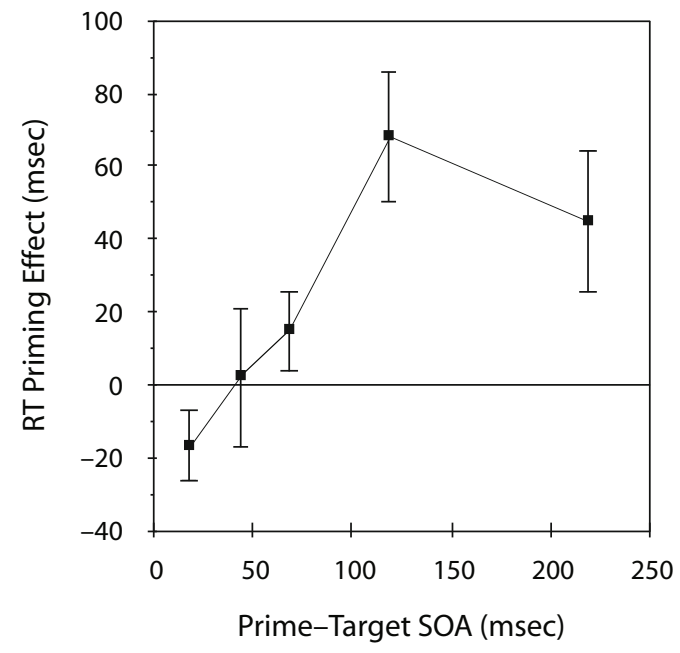

Figure 4. Priming function: Priming effects in the response time (RT) condition calculated as the RT difference between incongruent and congruent trials as a function of the stimulus onset asynchrony (SOA) between prime and target.

was followed by the target stimulus, prime discrimination performance was associated with a larger RT and disambiguation priming effects.

\section{DISCUSSION}

The major new finding of the present study is the demonstration of directional motion priming with a prime that rotated so rapidly that observers could not discriminate its motion direction. When it was presented by itself, the prime appeared to be a simultaneously flashed outline circle, and no observer that was tested could consciously discriminate its rotation direction. Since the P15 phosphor we used has virtually no visible persistence (Groner, Groner, Müller, Bischof, \& diLollo, 1993), this perceived simultaneity must be attributed to neural persistence. This persistence could have occurred at least in part at a level as early as the retina, although postretinal loci of persistence are also possible (Coltheart, 1980). However, when a target with an evident apparent rotation followed this prime, the choice RT to report the target direction was generally faster (and error rates were lower) when the prime and target motions were congruent than when they were incongruent. Thus, despite its phenomenal invisibility, information regarding the direction of the prime's spin must have been encoded by the retina and preserved at some subsequent visual-processing stages. As noted in the introduction, research has shown that grating stimuli with spatial frequencies too high to be consciously recognized can nevertheless generate tilt aftereffects (He \& MacLeod, 2001; Rajimehr, 2005) and generate distinguishable patterns of V1 activity (Haynes \& Rees, 2005), and that early visual cortex can respond to both chromatic (Gur \& Snodderly, 1997; Vul and MacLeod, 2006) and achromatic (Williams et al., 2004) flicker rates, which are not perceptually visible. The present findings suggest that motions with speeds too high to be consciously discriminated are likewise represented in early visual cortex.

\section{Motion Priming Compared to Shape and Color Priming}

Our use of an RT measure to assess motion priming permits direct comparisons with the shape-priming literature. Such comparisons are of interest because in the macaque, brain visual stimuli are processed by two distinct visual steams (DeYoe \& Van Essen; 1988 Ungerleider \& Mishkin, 1982), a ventral "what" pathway that processes shape and color and a dorsal "where" pathway that processes location and motion information. Human brain imaging studies have provided substantial evidence of a corresponding functional division in humans (Cohen et al., 1996; Goebel, Khorram-Sefat, Muckli, Hacker, \& Singer, 1998; Kourtzi, 2004; Taylor et al., 2000). However, the dynamic of the time course of the RT priming that we found matches that found with shape and color stimuli, both with reportable and metacontrast masked primes (Mattler, 2003; Schmidt, 2002; Vorberg et al., 2003, 2004). Specifically, we found that in the first $119 \mathrm{msec}$, priming increases monotonically with increasing SOA with a slope close to unity, so that the increase in the SOA approximately matches the RT reduction that is due to prime-target congruence (Figure 4). The generality of this distinctive outcome is commensurate with the view that the same mechanism may be responsible for the priming of RTs with shape, color, and motion stimuli. Should this be the case, it would imply that the system that mediates this priming is subsequent to the point where the ventral and dorsal systems converge. Intraparietal cortex is one candidate region where this could occur, since although it is generally regarded as a part of the dorsal stream, this region can respond to shape (Sereno \& Maunsell, 1998) and color (Toth \& Assad, 2002) information. Vorberg et al. (2003) have proposed a model designed to explain the unity gain slope in the case of shape priming, whereas other models predict priming functions with a slope close to 2 . These authors posit that the priming occurs at the level where decisions link sensory information to actions. The present study indicates that this model may also be applicable to motion priming.

Summing up, we found motion priming effects on RTs that are comparable to shape or color priming effects with respect to their (1) monotonic time course, (2) unity slope, and (3) the fact that they can occur irrespective of prime visibility. A parsimonious account of these RT priming effects would therefore posit that these different priming effects are produced by a single mechanism that receives information from sources in both the dorsal and ventral pathways.

\section{Initial Reverse Priming}

In the choice-RT task, the direction of the priming effect was reversed at the shortest $(19 \mathrm{msec})$ SOA: Responses were faster when the prime and target rotations were in opposite directions. Reverse motion priming has also been reported with a disambiguation measure when the priming 

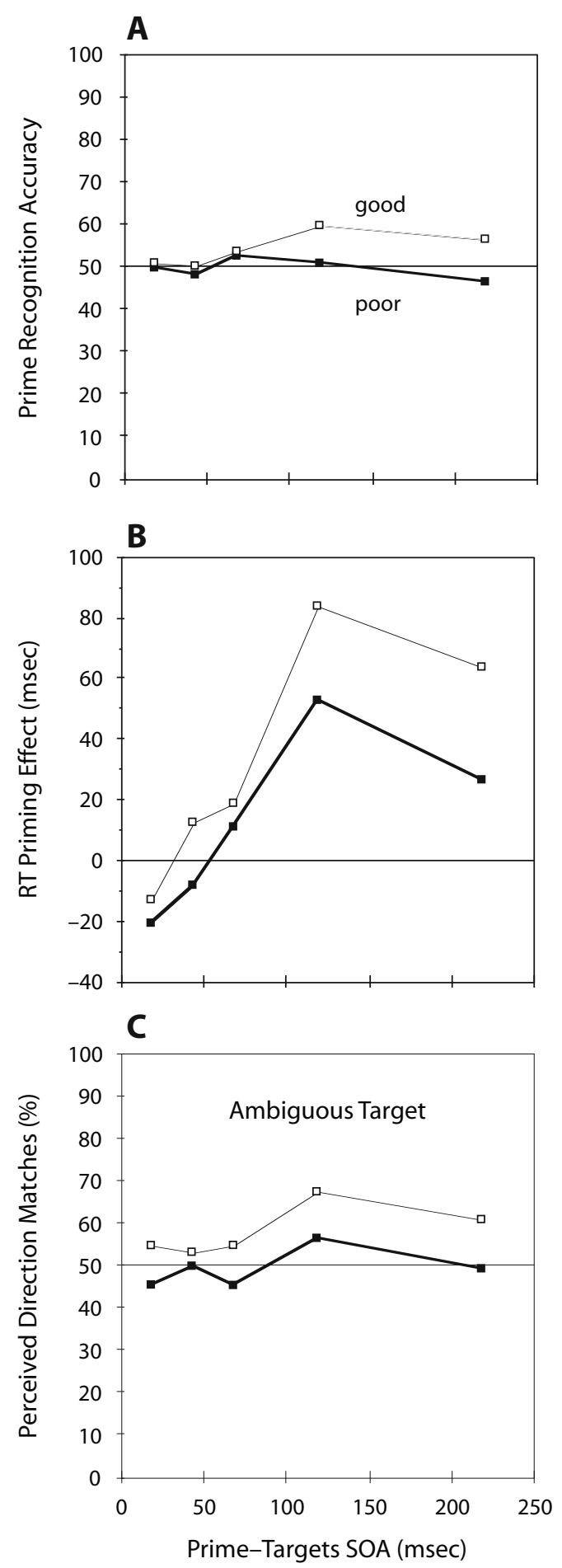

Figure 5. Prime discrimination performance and priming effects as a function of the stimulus onset asynchrony (SOA) in participants with good and poor prime identification performance in the prime direction condition. (A) Prime discrimination performance of five poor prime observers (filled symbols) and five good observers (open symbols) in the prime + discrimination condition. (B) Priming effects in the RT condition calculated as the RT difference between incongruent and congruent trials for poor (filled symbols) and good observers (open symbols). (C) Priming effects in the ambiguous motion condition for poor (filled symbols) and good observers (open symbols). stimulus has multiple apparent motion steps (Pantle et al., 2000). Piehler and Pantle (2001) proposed that this finding might be explained by a decrease in the sensitivity of mechanisms tuned to motion in the direction of the prime. Consistent with this hypothesis, they reported that brief apparent motion stimuli can reduce observers' abilities to detect subsequent apparent motion stimuli with the same direction when the stimuli are spatially overlapping. In our data, it is possible that a similar reduction in sensitivity slowed the processing of target motion congruent with the motion of the prime over the entire range of SOAs investigated. According to this view, the resulting delay in the processing of stimuli congruent with the prime direction increases all the RTs for the congruent trials (see Figure $3 \mathrm{~B}$ ). Since the priming function (Figure 4 ) reflects the RT difference between incongruent and congruent trials, the delay on congruent trials leads to a negative offset of the entire priming function, producing its negative intercept. The null effect with the 44-msec SOA would thus be attributable to a mutual cancellation of the sensory delay and the priming effect. However, this is a speculation that remains to be tested.

\section{Disambiguation Priming}

In previous studies, motion priming has been assessed by the tendency of an ambiguous apparent motion target to be seen as moving in the same direction as a preceding prime (Anstis \& Ramachandran, 1987; Blake et al., 1999; Pantle et al., 2000; Piehler \& Pantle, 2001; Pinkus \& Pantle, 1997; Ramachandran \& Anstis, 1983). We found that our priming stimulus was also able to produce such disambiguation priming. However, although the RT priming effects that we observed spanned the full range of the SOAs that we tested, we observed disambiguation priming only with the 119-msec and (to a lesser extent) 219 -msec SOAs. Because the $119 \mathrm{msec}$ is the SOA in which priming was maximal with the RT measure, it is possible that these different patterns of priming simply reflect a lower sensitivity of the disambiguation measure. That is, because of its relative insensitivity, disambiguation may only have been capable of revealing priming effects when they were most robust. Alternatively, the different patterns of priming for RTs and disambiguation may reflect a difference in the systems mediating these two cases of priming.

\section{Effect of the Target on Discrimination of the Prime}

Although all subjects were at chance at judging the prime direction when it was presented by itself (the primeonly discrimination condition), when the target followed the prime, some participants were able to report the prime direction with above-chance accuracy (the prime + discrimination condition). This ability was most pronounced at the 119-msec SOA, and at this SOA, it reached significance across participants. This instance of nonchance prime discrimination performance occurring at the same SOA in which we observed the strongest RT priming effects, along with its association with disambiguation priming, lead us to think that it is a genuine phenomenon. 
In this case, the data suggest that an interaction between the prime and target at this SOA sometimes allowed subjects to become aware of the prime's rotation direction. This interaction could have either enhanced the motion information conveyed by the prime, making that motion discernible, or provided a cue to the prime's direction by altering the appearance of the target. These possibilities cannot be distinguished on the basis of the present experiments, but data recently collected in our lab on consciousness mediated by neural transition states (manuscript in preparation).

\section{Potential Contributions of Awareness}

When we divided participants into two groups on the basis of their performance in the prime + discrimination condition, only the better of the two groups showed a better-than-chance ability to discriminate the prime direction (Figure 5A). This group also showed significantly stronger RT priming effects. However, the RT priming effects were significant in both groups and followed the same pattern as the SOA was varied (Figure $5 \mathrm{~B}$ ). If the above-chance prime discrimination performance reflected an increased awareness of the prime direction, these outcomes suggest that awareness was not needed to produce the RT priming we observed, but could act to facilitate that priming. Disambiguation priming was also stronger in the group with better prime-discrimination performance. However, the group that performed poorly in the prime + discrimination condition performed at or below chance in the disambiguation condition at every SOA except $119 \mathrm{msec}$ (Figure 5C), whereas RT priming effects were evident in this group at multiple SOAs. This result leads us to speculate that the contribution of awareness may have more importance for disambiguation priming than for RT priming. This speculation is commensurate with primate single-cell data reported by Williams, Elfar, Eskandar, Toth, and Assad (2003), who found that activity in LIP cells predicted the perception of an ambiguous motion stimulus before the onset of the stimulus motion. This finding suggests that the disambiguation of motion percepts might be at least partially dependent on processing at a high level in the dorsal motion system that is linked to awareness of the prime.

On the other hand, Blake et al. (1999) found disambiguation priming with primes that were presented to an eye in which conscious vision was suppressed during binocular rivalry, and at the 119-msec SOA, we did observe a small but significant disambiguation priming effect in the group that performed poorly in the prime + discrimination condition. Therefore, we cannot conclude from our data that there is there is a complete dependence of disambiguation priming on awareness. Moreover, it remains possible that the above-chance prime-discrimination performance in the prime + discrimination condition was not produced by an actual perception of the prime's rotation direction, but by a cue provided by the effect of the prime on the subsequent target. Nevertheless, we think additional research to establish the extent to which the role of awareness is different for disambiguation and RT priming would be worthwhile.

\section{Neural Correlates}

The present findings suggest that rapid motions that cannot be consciously discriminated are represented in human visual cortex. If the information coding the rotation direction of the prime follows the normal route of liminal motion information, it projects from the retina to V1 via the LGN and then via the prestriate cortex on to motion-processing area MT. The possibility that the motion information in our primes is processed by $\mathrm{V} 1$ gains credibility from the finding that in macaques, some V1 neurons can follow heterochromatic flicker well beyond the perceptual fusion frequency, and luminance flicker at rates near the human luminance fusion point (Gur \& Snodderly, 1997). However, there are alternative pathways from the retina to MT that bypass V1 (Barbur, Watson, Frackowiak, \& Zeki, 1993; Schoenfeld, Heinze, \& Woldorff, 2002; Schoenfeld; Noesselt, et al., 2002; Sincich, Park, Wohlgemuth, \& Horton, 2004; Stoerig \& Cowey, 1997). Future research is needed to determine the specific stages in the visual processing hierarchy in which the spin of our prime had a neural representation, and the stages in which that representation is lost. Imaging techniques such as fMRI should prove useful in this regard.

\section{AUTHOR NOTE}

We thank Susan Meißner for collecting the data. Correspondence concerning this article should be addressed to U. Mattler, Georg-Elias-Müller Institute for Psychology, Georg August University Göttingen, Gosslerstrasse 14, D-37073 Göttingen, Germany (e-mail: uwe.mattler@psych uni-goettingen.de).

\section{REFERENCES}

ANSTIS, S. M., \& RAMACHANDRAN, V. S. (1987). Visual inertia in apparent motion. Vision Research, 27, 755-764.

Barbur, J. L., Watson, J. D., Frackowiak, R. S., \& Zeki, S. (1993). Conscious visual perception without V1. Brain, 116, 1293-1302.

BLAKE, R. (2001). A primer on binocular rivalry, including current controversies. Brain \& Mind, 2, 5-38.

Blake, R., Ahlstrom, U., \& Alais, D. (1999). Perceptual priming by invisible motion. Psychological Science, 10, 145-150.

Blake, R., \& Logothetis, N. K. (2002). Visual competition. Nature Review Neuroscience, 3, 13-21.

Breitmeyer, B. G. (1984). Visual masking. An integrative approach. Oxford: Oxford University Press.

Cohen, M. S., Kosslyn, S. M., Breiter, H. C., DiGirolamo, G. J., Thompson, W. L., Anderson, A. K., ET AL. (1996). Changes in cortical activity during mental rotation. Brain, 119, 89-100.

Coltheart, M. (1980). Iconic memory and visible persistence. Perception \& Psychophysics, 27, 183-228.

DeYoe, E. A., \& Van Essen, D. C. (1988). Concurrent processing streams in monkey visual cortex. Trends in Neurosciences, 11, 219-226.

DiLollo, V., Enns, J. T., \& Rensink, R. A. (2000). Competition for consciousness among visual events: The psychophysics of reentrant visual processes. Journal of Experimental Psychology: General, 129, 481-507.

Eimer, M., \& Schlaghecken, F. (1998). Effects of masked stimuli on motor activation: Behavioral and electrophysiological evidence. Journal of Experimental Psychology: Human Perception \& Performance, 24, 1737-1747.

Fehrer, E., \& RAAB, D. (1962). Reaction time to stimuli masked by metacontrast. Journal of Experimental Psychology, 63, 143-147.

Goebel, R., Khorram-Sefat, D., Muckli, L., Hacker, H., \& Singer, W. (1998). The constructive nature of vision: Direct evidence from functional magnetic resonance imaging studies of apparent motion and motion imagery. European Journal of Neuroscience, 10, 1563-1573. 
Groner, R., Groner, M. T., Müller, P., Bischof, W. F., \& DiLollo, V. (1993). On the confounding effects of phosphor persistence in oscilloscopic displays. Vision Research, 33, 913-917.

Gur, M., \& SNODDERLY, D. M. (1997). A dissociation between brain activity and perception: Chromatically opponent cortical neurons signal chromatic flicker that is not perceived. Vision Research, 37, 377-382.

Haynes, J.-D., \& Rees, G. (2005). Predicting the orientation of invisible stimuli from activity in human primary visual cortex. Nature Neuroscience, $\mathbf{8}, 686-691$.

He, S., \& MacLeod, D. I. A. (2001). Orientation-selective adaptation and tilt after-effect from invisible patterns. Nature, 411, 473-476.

Klotz, W., \& Neumann, O. (1999). Motor activation without conscious discrimination in metacontrast masking. Journal of Experimental Psychology: Human Perception \& Performance, 25, 976-992.

Kourtzi, Z. (2004). 'But still, it moves.' Trends in Cognitive Sciences, $\mathbf{8}, 47-49$.

Lleras, A., \& EnNs, J. T. (2004). Negative compatibility or object updating? A cautionary tale of mask-dependent priming. Journal of Experimental Psychology: General, 133, 475-493.

Loftus, G. R., \& Masson, M. E. J. (1994). Using confidence intervals in within-subject designs. Psychonomic Bulletin \& Review, 1, 476490.

MackNiK, S. L., \& LivingStone, M. S. (1998). Neural correlates of visibility and invisibility in the primate visual system. Nature Neuroscience, 1, 144-149.

Marcel, A. J. (1983). Conscious and unconscious perception: Experiments on visual masking and word recognition. Cognitive Psychology, 15, 197-237.

MattLer, U. (2003). Priming of mental operations by masked stimuli. Perception \& Psychophysics, 65, 167-187.

MatTLER, U. (2005). Inhibition and decay of motor and nonmotor priming. Perception \& Psychophysics, 67, 285-300.

MatTler, U. (2006). On the locus of priming and inverse priming effects. Perception \& Psychophysics, 68, 975-991.

McCollough, C. (1965). Adaptation of edge-detectors in the human visual system. Science, 149, 1115-1116.

Milliken, B., Joordens, S., Merikle, P. M., \& Seiffert, A. E. (1998). Selective attention: A reevaluation of the implications of negative priming. Psychological Review, 105, 203-229.

Neumann, O., \& KLOTZ, W. (1994). Motor responses to nonreportable masked stimuli: Where is the limit of direct parameter specification? In C. Umiltà \& M. Moscovitch (Eds.), Attention and performance XV: Conscious and nonconscious information processing (pp. 124-150). Cambridge, MA: MIT Press.

Pantle, A. J., Gallogly, D. P., \& Piehler, O. C. (2000). Direction biasing by brief apparent motion stimuli. Vision Research, 40, 19791991.

Piehler, O. C., \& Pantle, A. J. (2001). Direction-specific changes of sensitivity after brief apparent motion stimuli. Vision Research, 41 , 2195-2205.

Pinkus, A., \& PantLe, A. (1997). Probing visual motion signals with a priming paradigm. Vision Research, 37, 541-552.

RAJIMEHR, R. (2005). Unconscious orientation processing. Neuron, 41, 663-673.

RAMACHANDRAN, V. S., \& ANSTIS, S. M. (1983). Extrapolation of motion path in human visual perception. Vision Research, 23, 83-85.

Schmidt, T. (2000). Visual perception without awareness: Priming responses by color. In T. Metzinger (Ed.). Neural correlates of consciousness: Empirical and conceptual questions (pp. 157-169). Cambridge, MA: MIT Press.

SchmidT, T. (2002). The finger in flight: Real-time motor control by visually masked color stimuli. Psychological Science, 13, 112-118.

Schoenfeld, M. A., Heinze, H.-J., \& WoldorfF, M. G. (2002). Unmasking motion-processing activity in human brain area V5/MT+ mediated by pathways that bypass primary visual cortex. Neuroimage, 17, 769-779.

Schoenfeld, M. A., Noesselt, T., Poggel, D., Tempelmann, C.,
Hopf, J.-M., WoldorfF, M. G., ET AL. (2002). Analysis of pathways mediating preserved vision after striate cortex lesions. Annals of Neurology, 52, 814-824.

Sereno, A. B., \& Maunsell, J. H. R. (1998). Shape selectivity in primate lateral intraparietal cortex. Nature, 395, 500-503.

Sincich, L. C., Park, K. F., Wohlgemuth, M. J., \& Horton, J. C. (2004). Bypassing V1: A direct geniculate input to area MT. Nature Neuroscience, 7, 1123-1128.

Stoerig, P., \& Cowey, A. (1997). Blindsight in man and monkey. Brain, 120, 535-559.

Taylor, J. G., Schmitz, N., Ziemons, K., Grosse-Ruyken, M.-L., Gruber, O., Hueller-Gaertner, H.-W., et AL. (2000). The network of brain areas involved in the motion aftereffect. NeuroImage, 11, 257-270

Tong, F. (2001). Competing theories of binocular rivalry: A possible resolution. Brain \& Mind, 2, 55-83.

Tong, F. (2003). Primary visual cortex and visual awareness. Nature Reviews Neuroscience, 4, 219-229.

Tотн, L. J., \& Assad, J. A. (2002). Dynamic coding of behaviourally relevant stimuli in parietal cortex. Nature, 415, 165-168.

UnGerLeIDER, L. G., \& MishKIN, M. (1982). Two cortical visual systems. In D. B. Ingle, M. A. Goodale, \& R. J. Q. Mansfield (Eds.), Analysis of visual behavior (pp. 549-586). Cambridge, MA: MIT Press.

Vorberg, D., Mattler, U., Heinecke, A., Schmidt, T., \& SchwarzBACH, J. (2003). Different time courses for visual perception and action priming. Proceedings of the National Academy of Sciences, 100, 6275-6280

Vorberg, D., Mattler, U., Heinecke, A., Schmidt, T., \& SchwarzBACH, J. (2004). Invariant time course of priming with and without awareness. In C. Kaernbach, E. Schröger, \& H. Müller (Eds.), Psychophysics beyond sensation. Laws and invariants of human cognition (pp. 271-288). Mahwah, NJ: Erlbaum.

Vul, E., \& MacLeod, D. I. A. (2006). Contingent aftereffects distinguish conscious and preconscious color processing. Nature Neuroscience, 9, 873-874.

Watanabe, T., Nanez, J. E., \& Sasaki, Y. (2001) Perceptual learning without perception. Nature, 413, 844-848.

Williams, P. E., Mechler, F., Gordon, J., Shapley, R., \& Hawken, M. J. (2004). Entrainment to video displays in primary visual cortex of macaque and humans. Journal of Neuroscience, 24, 8278-8288.

Williams, Z. M., Elfar, J. C., Eskandar, E. N., Toth, L. J., \& Assad, J. A. (2003). Parietal activity and the perceived direction of ambiguous apparent motion. Nature Neuroscience, 6, 616-623.

WolfF, P. (1989). Einfluss des maskierten Testreizes auf die Wahlreaktion auf den Maskierreiz bei Metakontrast [Effect of masked stimulus on choice response to the masking stimulus in metacontrast]. Paper presented at the 31st Tagung experimentell arbeitender Psychologen, Bamberg, Germany.

\section{NOTE}

1. A signal detection analysis of the data in the 119-msec condition showed similar results in the two conditions: $d^{\prime}$ values differed significantly from zero in the disambiguation condition $\left[d^{\prime}=0.73, t(9)=\right.$ $4.8, p=.001]$ and in the prime + discrimination condition $\left[d^{\prime}=0.37\right.$, $t(9)=3.3, p=.01]$, and there was no significant effect on response bias measure beta in either condition $[t(9)<1.4, p>.22$ in each case]. Individual $t$ tests at each level of SOA indicated that there were no significant values of beta at any level of SOA in the disambiguation condition $[t(9)<1.0, p>.40]$. A significant value of beta did occur with the 69-msec SOA in the prime + discrimination condition, but this effect does not survive a Bonferroni correction $[t(9)=2.6, p=.32 ; t(9)<$ $1.4, p>.22$ in all other cases]. In general, this analysis is commensurate with the possibility of a link between the above-chance performances in the prime + discrimination and disambiguation tasks.

(Manuscript received April 20, 2006; revision accepted for publication May 22, 2007.) 